\title{
Arrogance in Classification
}

\author{
Amy L. Magnus \\ Design and Assessment Technology \\ Defense Threat Reduction Agency \\ 6801 Telegraph Road \\ Alexandria, VA, USA 22310 \\ (703)325-6622 \\ Amy.Magnus@dtra.mil (or clevergirl@alum.rit.edu)
}

\author{
Mark E. Oxley \\ Department of Mathematics and Statistics \\ Air Force Institute of Technology \\ 2950 P Street \\ Wright-Patterson AFB, OH, USA 45433-7765 \\ (937)225-3636 \\ Mark.Oxley@afit.edu
}

\begin{abstract}
The Department of Defense and the Department of the Air Force have funded automatic target recognition for several decades with varied success. The foundation of automatic target recognition is based upon pattern recognition. In this paper, we present new pattern recognition conceptsspecifically in the area of classification-and propose a new visualization technique that allows one to determine when a classifier is being arrogant. Clearly, arrogance in classification is an undesirable attribute. A human is being arrogant when their expressed conviction in a decision overstates their actual experience in making similar decisions. Likewise, given an input feature vector we say a classifier is arrogant in its classification if its veracity is high yet its experience is low. Conversely, a classifier is non-arrogant in its classification if there is a reasonable balance between its veracity and its experience. It is possible to quantify this balance and we discuss new techniques that will detect arrogance in a classifier.
\end{abstract}

We show that single-hidden layer perceptrons are arrogant in their classification of certain regions in a feature space. When a classifier has been optimized to perform well on truthed data, one hopes that the classifier will perform well on operational (unknown) data. If a classifier performs well on new, previously-unseen data then we say that the classifier generalizes well. Quantifying this generalization performance has several approaches, as the literature shows, including holdout methods, boosting and bagging. In a departure from these stochastic approaches, we use 4-value logic to quantify generalization performance. Four-value logic is a simplified expression of both veracity and experience. For a multilayer perceptron (MLP), we detail where in feature space the classifier is arrogant and where it is non-arrogant. Our analysis yields new evaluation tools for the MLP and paves the way for other classifiers. The specific tools for the multilayer perceptron are based upon combinatorial geometry techniques that have existed for more than a decade, but are just now being exploited in the computational intelligence community.

The benefits of our 4-value logic technique over other iterative and stochastic methods include the following: (1) Our technique evaluates the generalization of an MLP after model

The views expressed in this article are those of the authors and do not re flect the official policy or position of the United States Air Force, Department of Defense, or the US Government

U.S. Government work not protected by U.S. copyright.

IEEEAC paper \# 1405 selection, requiring only the classifier's weights and biases and the data used to train these parameters. Iterative methods require that the evaluation of generalization take place over multiple training runs prior to the selection of a specific model; then, once a model is selected, the estimation of generalization for the selected model is optimistic. (2) The results of our evaluation do not change based on the order that data are presented. (3) Stochastic techniques exercise very little use of the domain, that is, the measure of the set of feature vectors used is zero. For our technique, the measure of such sets used is positive.

\section{TABLE OF CONTENTS}

\author{
1 INTRODUCTION \\ 2 EXPERTISE LOGIC AND ARROGANT CLASSIFIERS \\ 3 THE ARROGANT MULTILAYER PERCEPTRON \\ 4 CONCLUSION
}

\section{INTRODUCTION}

This work is part of a larger research effort [1] addressing the hard problem [2] in computational intelligence: selfevaluation in machines. The immediate goal of our research is the quantification of experience in classification applications. Quantifying experience allows us to separate expert classifiers from "pretenders". A classifier is an explicit relation designed from labeled training data and extended to the larger set of unlabeled data collected in the operational use of the relation. An expert is a special classifier that has a quantifiable skill level. Expert classifiers may be too bold or too timid in extending their training data to the larger operational set. Traditionally, we judge classifiers by assessing the amount of apparent memorization in classification. When a classifier memorizes-or overfits-training data, it is too timid in associating data near training data with the training data's label. Classifiers may also be too bold in assigning labels to data far from training data. We call this arrogance in classification. Arrogance is an over-statement of expertise where a classifier has no or insufficient experience. We define arrogance in classification formally in Section 2 and demonstrate an arrogant classifier-the multilayer perceptron-in Section 3 . 


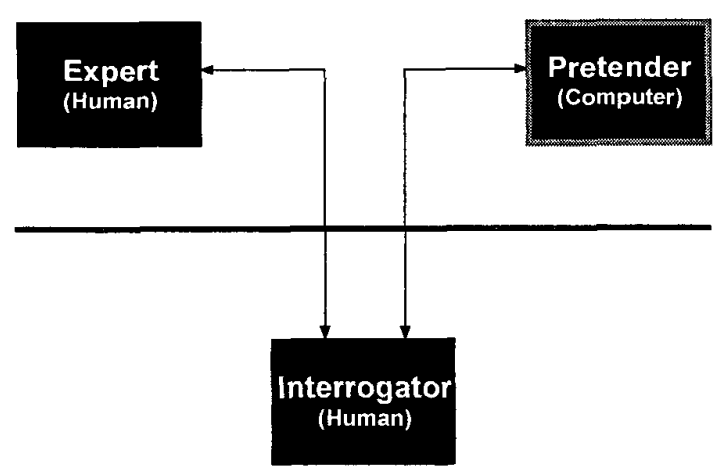

Figure 1. Turning's test for computer intelligence. If a computer is able to convince an interrogator that it is human, Turing suggested that this is a sign of intelligence.

\section{Turing and the intelligent pretender}

Alan Turing introduced the concept of "computer as pretender" in a 1950 article "Computing Machinery and Intelligence" [3]. In this article, he presented a test for computer intelligence that has become known as the Turing Test. Turing proposed an imitation game in which, if a computer can fool a human interrogator, then the computer is intelligent. Imagine a game played by three people: a man, a woman, and an interrogator. The interrogator stays in a room apart from the other two but is allowed to submit questions to them via a messaging system. The interrogator's objective is to determine who is the woman. The role of the woman is to be the expert and to convince the interrogator that she is, indeed, the woman. The role of the man is to be the pretender-that is, to fool the interrogator that he is the woman. In the Turing Test, Turing proposed replacing the pretender with a computer-as seen in Figure 1-and suggested that an intelligent computer would be able to fool the human interrogator that it is also human. [3]

One problem with the Turing Test is its subversive nature. It is ethically dubious to suggest that a computer must misrepresent itself in order to prove it is intelligent. We would prefer a computer capable of weeding out the pretender rather than a computer that aspires to be one. Consequently, we propose a modified Turing Test which we shall call the Inquisitive Test.

\section{Inquisitiveness in machines}

The Inquisitive Test, shown in Figure 2, asserts that a computer is inquisitive if it is able to distinguish an expert from a pretender. In our form of the imitation game, we replace the human interrogator with a computer while the expert and the pretender may be humans or computers. The Inquisitive Test-more so than the Turing Test-has real and immediate application to information management systems under development today. Military applications for quantifying expertise abound, particularly in systems involving technologies such

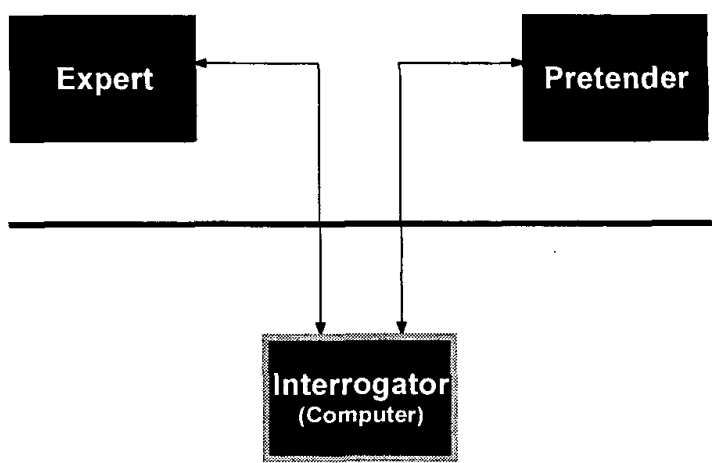

Figure 2. Our proposed test for inquisitiveness. We suggest that an inquisitive computer in its role as interrogator has the ability to distinguish an expert from a pretender. In the case where all three players-the interrogator, the expert and the pretender-are internal to an autonomous computer agent, the inquisitive test is an exercise in self-evaluation.

as Information Fusion, Intelligence Amplification ${ }^{1}$, and Multi Agent Systems. One example is a new initiative from the Air Force Office of Scientific Research, the Hybrid Inferencing from Fused Information (HIFI) program; the goal of this program is to replicate expertise in human intelligence gathering through automation and to create and maintain flexible ontologies shared between human and automated systems. Another Air Force application is sensor-based treaty monitoring where automated experts must confidently separate rare events from events that occur $1000+$ times a day.

Inquisitiveness is marked by a drive to bound experience. We see inquisitiveness in machines as a necessary step toward self-evaluation and true intelligence-that is, to say, an intelligent computer must be inquisitive, but the inquisitive computer is not necessarily intelligent. Instead, an inquisitive computer achieves a primitive stage of critical thinking analogous to the "Terrible $2 \mathrm{~s}$ "- the age where children are infamous for asking "Why? Why?" ad nauseam. This behavior is an attention-getting device, but the child is also acting on the recent revelation that their parents don't know everything. Children at this age aren't really interested in knowing exactly why the sky is blue; instead, they are attempting to bound the limits of what their parents know. If so motivated, this stage in child development is a striking early sign of critical thinking - the disciplined intellectual criticism that combines research, knowledge of historical context, and balanced judgment [5].

We believe the Inquisitive Test is an important intermediate demonstration of intelligence and, to the benefit of Artificial Intelligence research, far more easily realizable. Automated interrogators have application in the evaluation of relative expertise and the promotion of intelligence amplification among

\footnotetext{
${ }^{1}$ Intelligence Amplification (IA) is the enhancement of human intelligence through human-computer interaction [4].
} 


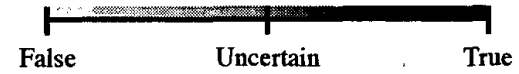

(a) Expert veracity

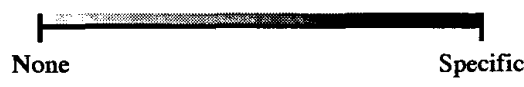

(b) Expert experience level

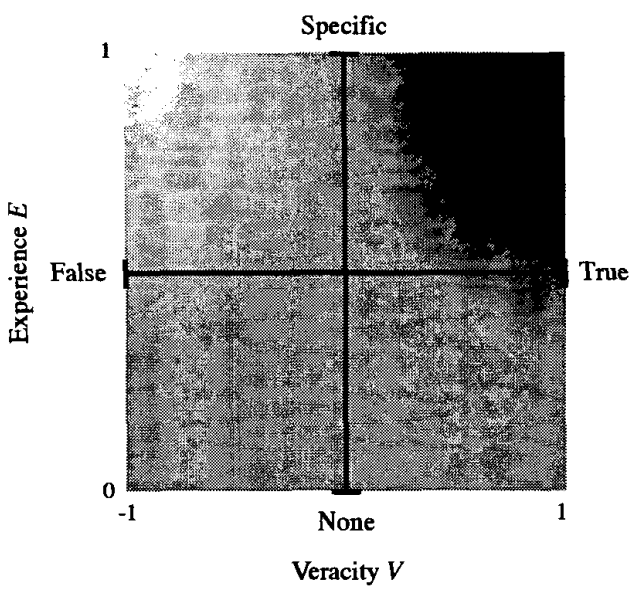

(c) Expert veracity versus experience

Figure 3. The uncertainty of an expert is quantified in two intervals: (a) Expert veracity, (b) Expert experience. Uncertainty is conceptualized as a function of grayscale on (c) the experience level versus veracity set.

experts. The advancement of scientific knowledge often turns on asking the right question. We can expect the advancement of machine-based knowledge to turn on the same point.

\section{Confusion in classification}

The success of a classifier hinges on its ability to interpolate operational data accurately. Success is measured by quantifying (1) the fitness of the classifier in ordering the hypothesized operational set and (2) the completeness of the training set in representing the operational set. When the operational set is largely untruthed, error measures-model versus truth comparisons-have limited utility. To compensate, we propose the use of confusion measures-model versus model comparisons- to evaluate expert classifiers over untruthed but interesting portions of the operational set.

Within the next section, we define the expert classifier and propose a figure of merit to judge appropriate expressions of expertise over the domain of such a classifier. We base our theory of confusion [6] on the premise that learning agents don't need to be authoritative experts in a task to be able to recognize confusion among themselves. For instance, students in a classroom can advise each others of subjects in a curriculum where retraining with additional resources and special care is needed. In other words, the students do not need to be experts in a subject to make reasonable judgements on whether their teachers' instruction needs to be augmented. We would like computer agents to be able to exhibit similar reasoning skills in self and peer-to-peer evaluation. Toward this end, we construct a confusion measure by contrasting a classifier's veracity to its apparent skill. We have deemed this construction expertise logic.

\section{EXPERTISE LOGIC AND ARROGANT CLASSIFIERS}

Expertise logic orders the opinions of experts. An expert is a special classifier-a relational computation with not only a mechanism for decision making but also a quantifiable skill level. We propose expertise logic as a means of conveying relative experience among experts and avoiding expressions of arrogance. Expertise logic is derived from the two quantities shown in Figures 3(a) and (b): veracity and experience. Next, we shall define these quantities and the concept of arrogance as they relate to classifiers.

\section{Veracity versus expertise}

First, let us define veracity and experience. Let $A$ be a classifier defined on feature set $\mathcal{X}$ with label set $\mathcal{L}$. Assume that $A$ was trained on subset $\mathcal{A} \subset \mathcal{X} \times \mathcal{L}$. We consider the pair $(A, \mathcal{A})$ together. Given a feature vector $x \in \mathcal{X}$ one wishes to construct a mapping $(x, A, \mathcal{A}) \rightarrow(\ell, \mathfrak{e}, \mathfrak{v})$ where the threetuple $(\ell, \mathfrak{e}, \mathfrak{v})$ represents the represents the expert's decision. Here $\ell \in \mathcal{L}$ is the label that the classifier $A$ produces. The output $e$ quantifies the experience associated with the input $x$ and training set $\mathcal{A}$. The output $\mathfrak{v}$ quantifies the veracity of the classifier towards its choice of the label $\ell$.

Veracity-Veracity ${ }^{2}$ is the classifier's internal conviction towards truthfulness of a label $\ell \in \mathcal{L}$.

Definition 1: (Veracity) The veracity of a classifier $A$ at feature $x$ is a quantity of the strength of conviction of the classification $\ell=A(x)$. Let $\mathfrak{v}=V_{A}(x) \in[0,1]$ denote the veracity of classifier $A$ at $x$. If $V_{A}(x)=0$ then the classifier has no conviction towards the chosen label $A(x)$. If $V_{A}(x)=1$ then the classifier is fully convicted towards its choice of the label.

Veracity must not be misinterpreted as a measure of correctness. A classifier may assign an incorrect label but maintain a strength of conviction towards this labeling. Given the 2class problem with label set $\mathcal{L}=\{$ false, true $\}$, veracity is mapped into two different intervals $V_{\text {false }}(x) \in[0,1]$ and $V_{\text {true }}(x) \in[0,1]$. For illustration purposes, we have depicted the two veracities in a single interval in Figure 3(a) by mapping $-V_{\text {false }}(x) \in[-1,0]$ and $V_{\text {true }}(x) \in[0,1]$ to form the interval $V(x) \in[-1,1]$.

Note if the conviction for a true classification is small, it does not necessarily follow that the classification is false. This is an important distinction because otherwise we assume the

\footnotetext{
${ }^{2}$ For motivation on veracity, see the paper by Alsing et al[7].
} 


\section{Vol. 5-2040}

classifier is always interpolating. To assume a classifier is always interpolating is to assume that if a feature is not near training data of one class then it is near training data of another class. This assumption leads to false positives.

Experience-The experience of a classifier $A$ at the feature vector $x$ depends on the data set used to train the classifier, that is, data $\mathcal{A}$.

Definition 2: (Experience) The experience of a classifier $A$ at feature $x$ is a quantity of closeness of feature $x$ to training data $\mathcal{A}$. Let experience $\mathfrak{e}=E_{A}(x) \in[0,1]$ denote the experience of classifier $A$ at $x$. If $E_{A}(x)=0$ then the classifier has no experience with data near $x$. If $E_{A}(x)=1$ then the classifier has all-knowing experience with data near $x$.

There are several ways to construct the experience quantifiers. We will give an example; but, to understand the concept of experience, let us start with some background material concerning metric spaces. Let $\rho$ be a metric defined on the feature set $\mathcal{X}$ so that $(\mathcal{X}, \rho)$ is a metric space. Recall the definition of a metric.

Definition 3: (Metric [8]). Let $\mathcal{X}$ be a nonempty set. A metric is a real-valued function $\rho$ defined on all of $\mathcal{X} \times \mathcal{X}$ such that the following properties hold true:

1. $\rho(x, y) \geq 0$ for all $x, y \in \mathcal{X}$. (nonnegativity)

2. $\rho(x, y)=\rho(y, x)$ for all $x, y \in \mathcal{X}$. (symmetry)

3. $\rho(x, y) \leq \rho(x, z)+\rho(z, y)$ for all $x, y, z \in \mathcal{X}$. (triangle inequality)

4. $\rho(x, y)>0$ if and only if $x \neq y$. (positive definiteness)

Definition 4: (Metric Space[8]). Let $\mathcal{X}$ be a nonempty set and let $\rho$ be a metric defined on $\mathcal{X}$, then the pair $(\mathcal{X}, \rho)$ is said to be a metric space.

Definition 5: (Ball). Let $(\mathcal{X}, \rho)$ be a metric space. An open ball, or open hypersphere, at $x$ with radius $r$ is defined to be the set

$$
\mathrm{B}(x, r)=\{y \in \mathcal{X}: \rho(x, y)<r\} .
$$

A closed ball is the set

$$
\overline{\mathrm{B}(x, r)}=\{y \in \mathcal{X}: \rho(x, y) \leq r\} .
$$

Definition 6: (Distance to a Set). Given a subset $\mathcal{S} \subset \mathcal{X}$ and a feature $x \in \mathcal{X}$, we define the distance from $x$ to $\mathcal{S}$ to be

$$
\operatorname{dist}(x, \mathcal{S})=\inf \{\rho(x, y): y \in \mathcal{S}\} \text {. }
$$

Let us discuss an example of quantifying the experience of a classifier in terms of its training data. Let $A$ be a classifier defined on $\mathcal{X}$ trained on data $\mathcal{A}$, and let the set of features from the data be denoted as

$$
\mathcal{A}_{1}=\{x \in \mathcal{X}:(x, \ell) \in \mathcal{A}\}
$$

the set of first components. Here are a few axioms that we can assume about experience.

Axiom 1: Given a feature $x \in \mathcal{X}$, if there are no training data near $x$, then the experience $E_{A}(x)$ is small (close to 0 ).

Axiom 2: Given a feature $x \in \mathcal{X}$, if there are training data near $x$, then the experience $E_{A}(x)$ is large (close to 1 ).

These two axioms say that $E_{A}(x)$ is inversely proportional to $\operatorname{dist}\left(x, \mathcal{A}_{1}\right)$.

Axiom 3: Given a feature $x \in \mathcal{X}$, if $x$ is a training datum, then experience $E_{A}(x)=1$.

Experience is a random variable whose distribution depends on the amount of data near $x$. Accordingly, we formulate these axioms.

Axiom 4: Given a feature $x \in \mathcal{X}$, if there are few training data near $x$, then the experience $E_{A}(x)$ is biased towards small but is not necessarily small.

Axiom 5: Given a feature $x \in \mathcal{X}$, if there are many training data near $x$, then the experience $E_{A}(x)$ is biased towards large but is not necessarily large.

These last two axioms say that $E_{A}(x)$ is proportional to $\operatorname{card}(\mathcal{A})$.

To construct an example of an experience mapping, first recall the definition of the density at $x$ with respect to $\mathcal{A}_{1}$ to be

$$
\operatorname{den}\left(x, \mathcal{A}_{1}\right)=\lim _{r \rightarrow 0^{+}} \frac{\operatorname{card}\left(\mathcal{A}_{1} \cap \mathrm{B}(x, r)\right)}{\operatorname{vol}(\mathrm{B}(x, r))} .
$$

Of course, for finite sets $\mathcal{A}_{1}$ with $x \notin \mathcal{A}_{1}$ then $\operatorname{den}\left(x, \mathcal{A}_{1}\right)=$ 0 . If $x \in \mathcal{A}_{1}$ then $\operatorname{den}\left(x, \mathcal{A}_{1}\right)=\infty$ since $\operatorname{card}\left(\mathcal{A}_{1} \cap\right.$ $\mathrm{B}(x, r)) \geq 1$ for all $r>0$. Therefore, we modify the definition to work for finite sets. Choose the radius to be $r_{x}=2 \operatorname{dist}\left(x, \mathcal{A}_{1}\right)$. Now determine the volume of the ball with radius $r_{x}$. We know that the set $\mathcal{A}_{1} \cap \mathrm{B}\left(x, r_{x}\right)$ is nonempty, hence the cardinality is nonzero. Consider

$$
\frac{\operatorname{card}\left(\mathcal{A}_{1} \cap \mathrm{B}\left(x, r_{x}\right)\right)}{\operatorname{vol}\left(\mathrm{B}\left(x, r_{x}\right)\right)}
$$

which approximates the density at $x$. But, this quantity is not bounded between 0 and 1, nor does it satisfy Axiom 3. We apply the function $z /(z+1)$ to force the values to lie in $[0,1]$ and be increasing. Hence, consider

$$
E_{A}(x)=\frac{\operatorname{card}\left(\mathcal{A}_{1} \cap \mathrm{B}\left(x, r_{x}\right)\right)}{\operatorname{card}\left(\mathcal{A}_{1} \cap \mathrm{B}\left(x, r_{x}\right)\right)+\operatorname{vol}\left(\mathrm{B}\left(x, r_{x}\right)\right)} .
$$

Observe that this satisfies Axiom 3, since if $x \in \mathcal{A}_{1}$ then $r_{x}=$ $2 \operatorname{dist}\left(x, \mathcal{A}_{1}\right)=0$, thus, $\operatorname{vol}\left(\mathrm{B}\left(x, r_{x}\right)\right)=0$ and $E_{A}(x)=1$. Also, the other axioms hold true by construction. 


\section{Vol. 5-2041}

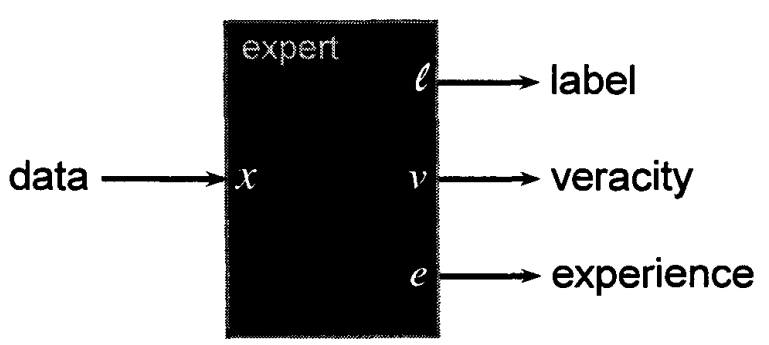

Figure 4. The expert classifier with a 3-tuple output representing a decision-the label $\ell$, a veracity score $\mathfrak{v}$ and a quantification of experience $\mathfrak{e}$-in relation to a given feature $x$.

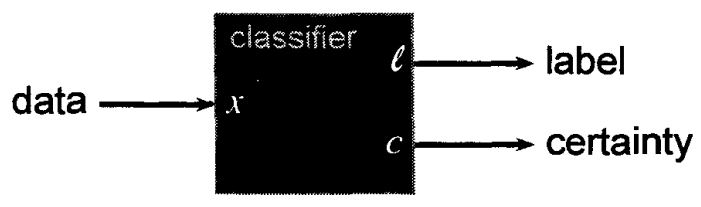

Figure 5. A classifier with a 2-tuple output representing a decision-the label $\ell$ and a confidence score $\mathfrak{c}$-in relation to a given feature $x$.

Mapping veracity versus experience - It is possible to map the veracity and experience measures of an expert classifier (per Figure 4) to a single measure of certainty c (per Figure 5). Unfortunately, there is significant expression lost in this reduction. Such a mapping is not one-to-one as several veracity-experience pairs map to a certainty of $c=0$. That is to say, there are many conditions where certainty $\mathfrak{c}=0$, and predominantly among of them are expressions of arrogance.

For each class $\ell \in \mathcal{L}$, we can relate a measure of certainty to corresponding veracities and experience. Figure 3 conceptualized this relation in veracity-experience space for the 2class problem. Given $\mathcal{L}=\{$ false, true $\}$, we mapped the veracity scores along the horizontal axis: The veracity $V_{\text {true }}(x)$ of the true class maps into the interval $[0,1]$, and the veracity of the false class maps into the interval $[-1,0]$ so that $-V_{\text {false }}(x) \in[-1,0]$. Let $\mathfrak{c}$ denote certainty. The range of certainty is depicted within bright-to-dark grayscale in Figure 3 (c) corresponding to the interval $[-1,1]$ so that "Certain False" $=-1$, "Uncertain" $=0$, and "Certain True" $=1$ respectively. The exact form of certainty $\mathfrak{c}(x)=\mathfrak{c}\left(V_{\ell}(x), E_{\ell}(x)\right)$ is domain dependent, but we can form the following axioms.

Axiom 6: Certainty $\mathfrak{c}$ approaches 0 as experience $\mathfrak{e}$ approaches 0 .

Axiom 7: Certainty $\mathfrak{c}$ approaches 1 as both veracity $\mathfrak{v}_{\text {true }} \rightarrow$ 1 and experience $\mathfrak{e}_{\text {true }} \rightarrow 1$ for class true.

Axiom 8: Certainty $c$ approaches -1 as both veracity $\mathfrak{v}_{\text {false }} \rightarrow 1$ and experience $\mathfrak{e}_{\text {false }} \rightarrow 1$ for class false.

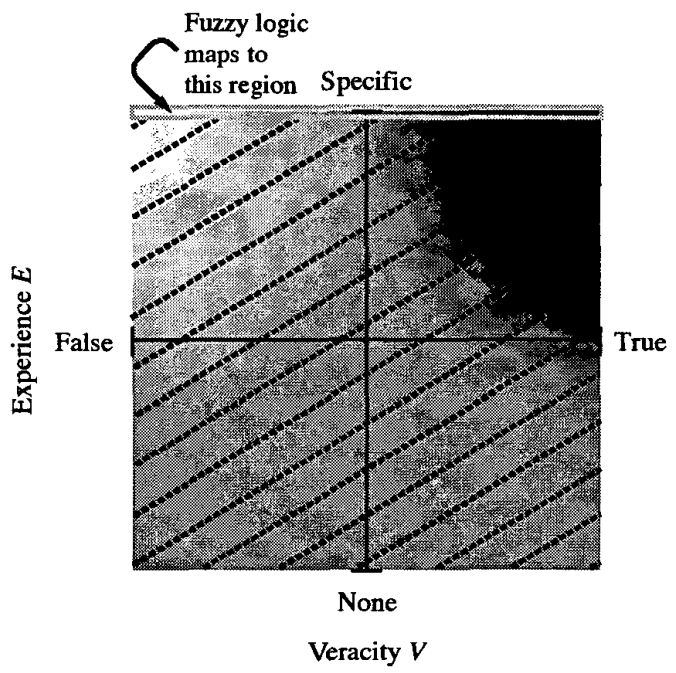

(a)

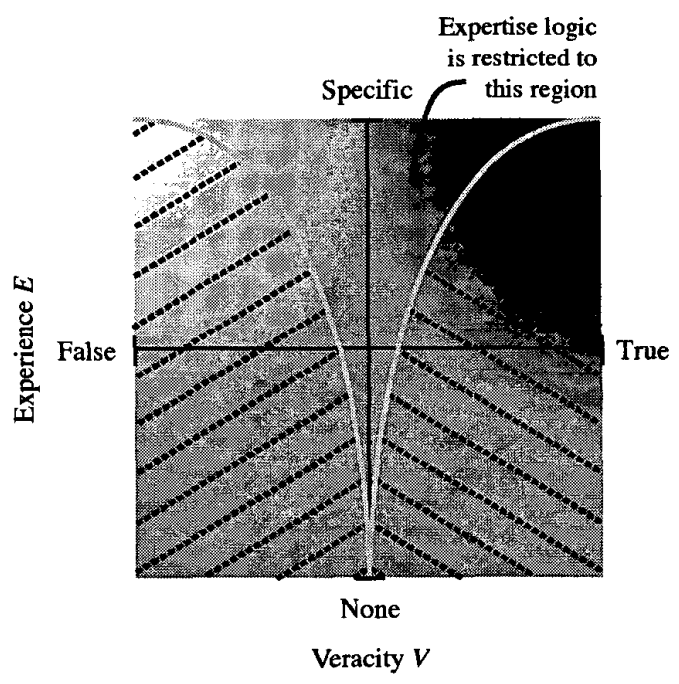

(b)

Figure 6. Mapping (a) fuzzy logic and (b) expertise logic onto the uncertainty set.

Following these axioms, we have conceptualized a generic form of certainty in Figure 3(c) such that certainty $c=0$ for the entire veracity interval where experience $\mathfrak{e}=0$, then $\mathfrak{c}=0$ for an increasingly smaller and smaller subinterval of the veracity interval as experience increases until, finally, $\mathfrak{c}=0$ is a point at experience $\mathfrak{e}=1$ and veracity $\mathfrak{v}=0$. When mapped into this conceptualization, we see fuzzy logic corresponds to the veracity interval where $\mathfrak{e}=1$ as shown in Figure 6(a). Thus, fuzzy logic presumes an expert encapsulates specific experience, and we conclude that fuzzy logic is most appropriate for data compression applications where this assumption holds true. 
When we project data generalizations onto the 2-dimensional veracity-experience set, care must be taken to map onto the "V" illustrated in Figure 6(b). This restriction in expertise logic ensures veracity does not overstate skill where there is little or no justification. As we shall demonstrate in Section 3 , the multilayer perceptron is a prime example of a classifier that tends to overstate experience. A single-hidden-layer MLP compactly represents high order logic by overly partitioning a feature space. Methods such as back propagation and cross validation do not regulate the ordering of partitions that do not contain training data. This leads to arbitrary and often misleading representations of veracity in regions where there are no training data. Where an unpopulated region is arbitrarily assigned a strong veracity $(\|\mathfrak{v}\| \rightarrow 1)$, we say the classifier expresses arrogance.

\section{Arrogance}

Some classifiers have an internal conviction toward certain answers without any collaborating experience. We call this arrogance. One must "trade-off" veracity versus experience before accepting a classifier's answer. We use an arrogance curve in veracity-experience space to do this.

Define a function $g:[0,1] \rightarrow[0,1]$ such that the following properties hold true:

(a) $g$ is defined on all of $[0,1]$;

(b) $g$ is non-decreasing on $[0,1]$;

(c) $g(0) \geq 0$

(d) $g(1) \leq 1$.

Examples of functions $g$ are:

1. $g(s)=s$;

2. $g(s)=\sqrt{s}$

3. $g(s)=s^{q}$ for some $q \in(0,1]$;

4. $g(s)=\log (s+1) / \log 2$;

5. $g(s)=\left\{\begin{array}{lll}0 & \text { for } & 0 \leq s<b \\ c & \text { for } & b \leq s \leq 1 .\end{array}\right.$

Let function $g$ define an arrogance curve such that, if experience $e$ is less than $g(\mathfrak{v})$ for veracity $\mathfrak{v}$, then the classifier expresses a certain amount of veracity without sufficient experience to back it up. The classifier is an arrogant classifier in this case. A decision maker should choose a threshold of arrogance appropriate to the application domain; thus, they choose a function $g$.

Definition 7: (Arrogant Classifier) Let $g$ be a function that satisfies the properties (a)-(d) above. Let $A: \mathcal{X} \rightarrow \mathcal{L}$ be a classifier with training set $\mathcal{A}$. Given a vector $x \in \mathcal{X}$ we say $A$ is arrogant at $x$ if $E_{A}(x)<g\left(V_{A}(x)\right)$.

Let us consider the arrogance curve given in Example 5 above. We use this curve in the construction of 4-value logic [9], a crisp-set interpretation of expertise logic. We use 4value logic to represent expertise logic in crisp sets in much

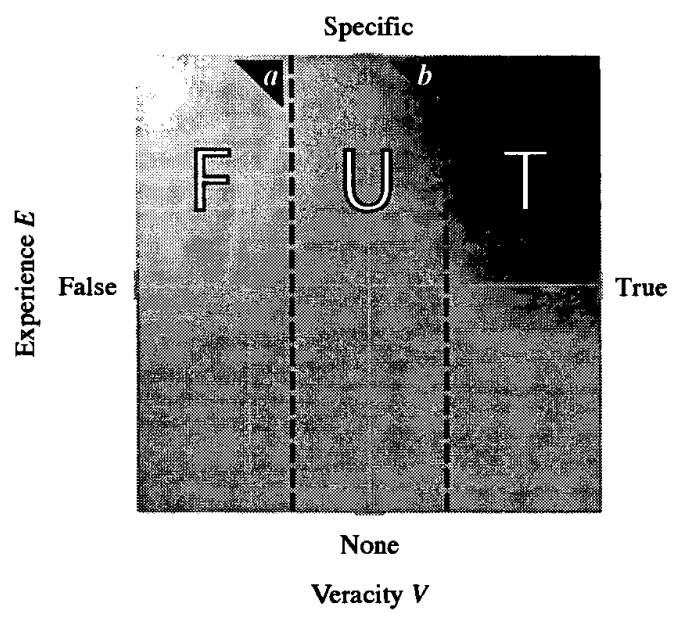

(a)

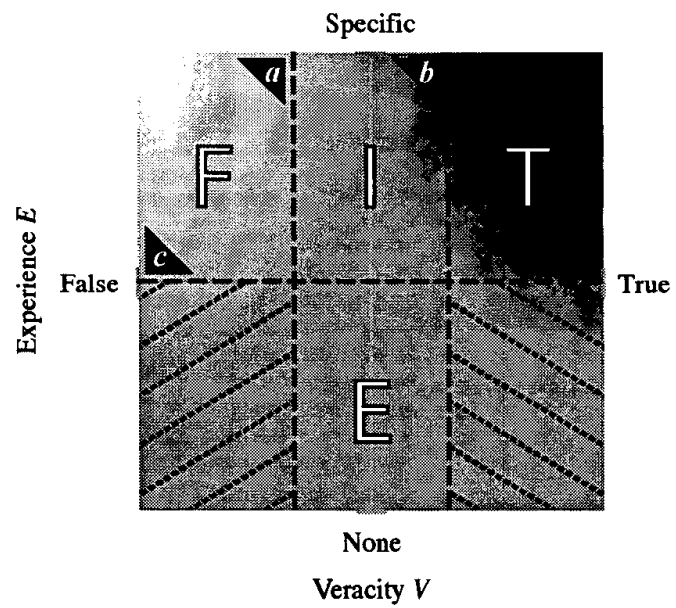

(b)

Figure 7. Three-value and four-value logic projected onto the uncertainty set. (a) Given two thresholds $a, b$, three-value logic divides the uncertainty set into 3 disjoint partitions. (b) Using three thresholds $a, b, c$, four-value logic divides the uncertainty set into 6 disjoint partitions: 4 subsets corresponding to 4-value logic and 2 subsets (the crossed out regions above) corresponding to arrogance.

the same way as 3-value logic represents fuzzy logic. In actuality, 4-value logic is a 6-value logic with two forbidden values. To explain, first examine fuzzy logic. Fuzzy logic maps expert opinions onto the interval $[0,1]$ and represents 3 -value logic as relaxed intervals: False $=[0, a]$, Uncertain $=(a, b)$, True $=[b, 1]$ where $a, b \in[0,1]$ and $a<b$ as shown in Figure 7(a). In a similar relaxed-interval treatment of 4-value logic, we construct expertise logic by mapping expert veracity versus experience. Given $a, b \in[-1,1]$ where $a<b$ and a third threshold $c \in[0,1], 4$-value logic is represented in the 
relaxed intervals

$$
\begin{aligned}
\text { False } & =[-1, a] \times[c, 1], \\
\text { True } & =[b, 1] \times[c, 1], \\
\text { Uncertain Interpolation } & =(a, b) \times[c, 1], \text { and } \\
\text { Extrapolation } & =[-1,1] \times[0, c] .
\end{aligned}
$$

The Extrapolation interval splits further into 3 partitions: $[-1, a] \times[0, c],(a, b) \times[0, c]$, and $[b, 1] \times[0, c]$. The second partition $(a, b) \times[0, c]$ expresses Uncertain Extrapolation (an appropriate expression) as shown in Figure 7(b); the first and third partition convey Arrogance. By forbidding a generalization to map into intervals that convey Arrogance, we restrict the mapping's output to 4-value logic: \{False, True, Uncertain Interpolation, Uncertain Extrapolation $\}$ or $\{f, t, i, e\}$.

\section{Reordering a domain in relation to expertise logic}

We assert that a good generalization isolates data, allowing extrapolations and uncertain interpolations to be easily separated from class data in a feature set. To illustrate good isolation, we recommend a figure of merit in which we order the membership of feature set in terms of the classifier's veracity scores and, then, cross reference this ordering to the classifier's experience scores. For good separation and appropriate expression of expertise, we prefer a classifier which orders its domain like Figure 8 . Note, it is desirable for features near "false" data points to score veracities near -1 and to have features near "true" points to score veracities near 1. To provide good isolation of classes, it is further desirable for feature near both "true" and "false" exemplars to score veracities approaching 0 (that is, along the non-linear transition between asymptotes) and for features far from any exemplar to score veracities of $\mathfrak{v}=0$.

Some classifiers merely separate training data from disparate classes and do not isolate the classes. These classifiers arbitrarily order extrapolated data (and may erratically order interpolated data depending on the classifier's complexity). In the arrogant classifier, significant regions of extrapolated regions receive veracity scores that are higher than scores assigned to truthed data. Instead of producing the desired domain ordering of Figure 8, the arrogant classifier orders the domain as depicted in Figure 9. We will demonstrate instantiations of such an ordering using a 4-node-input/3-nodeoutput multilayer perceptron in Section 3. For this demonstration, we chose an MLP trained using back propagation-a popular optimization technique-which separates class data without isolating the classes. Consequently, we expect the MLP to exhibit at least some degree of arrogance.

\section{Expertise logic summary}

Expertise logic gives us the degree of freedom to separate uncertain decisions based on vague experience from decisions based on no experience. For data generalizations, we must anticipate encountering regions of the domain where experience is incomplete and, in these regions, avoid making arrogant classifications. The power of expertise logic rests in the

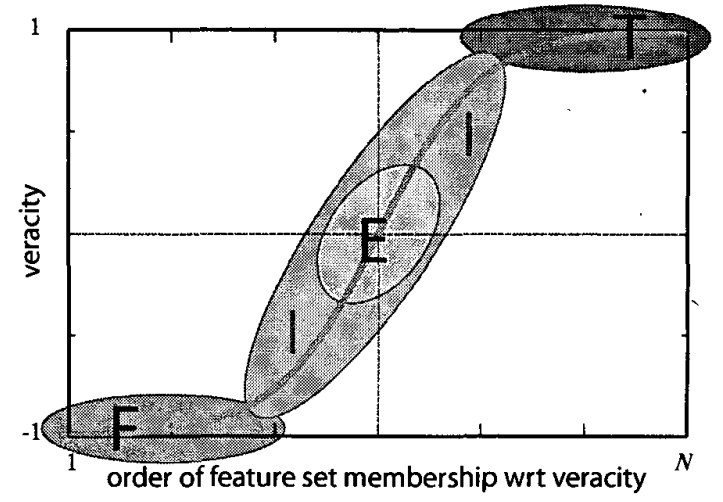

Figure 8. An appropriate expression of expertise: The desired clustering of experience based on the ordered veracity of a multilayer perceptron. Experience is represented in terms of 4-value logic. Specific experience should cluster in the appropriate asymptotic regions such where $\|\mathfrak{v}(x)\| \rightarrow 1$, while extrapolations should cluster tightly where $\mathfrak{v}(x)=0$.

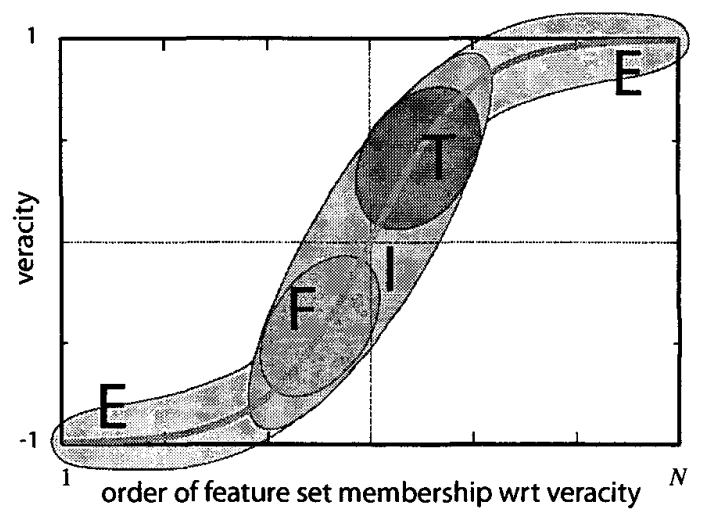

Figure 9. Expressions of arrogance: Our prediction of the clustering of experience based on the ordered veracity of an arrogant classifier. Extrapolations dominate the asymototic regions pushing specific experience away from the asymptotes toward $\mathfrak{v}(x)=0$.

assertion that a classifier embodies a hypothesis of what is considered near training data and what is considered far. By contrasting the hypotheses of multiple experts, feature space can be partitioned into regions of certainty, regions of uncertainty due to interpolation conflicts, and regions of high uncertainty due to extrapolation.

\section{THE ARROGANT MULTILAYER PERCEPTRON}

In this paper, we have selected a multilayer perceptron trained by back propagation as our example of an arrogant classifier. In general, we expect arrogant classification to occur in classifiers that separate but do not isolate data. For example, given a trained gaussian classifier for a multi-class problem, the implementation of a winner-take-all labeling scheme (without a 
rejection class) results in class assignment over the classifier's entire domain, even in extrapolated regions. To mitigate arrogance in classification, we advocated the use of isolation strategies which must strike a reasonable balance between being too timid and too bold in assigning class labels.

To demonstrate arrogance, we shall first define our measures that map a multilayer perceptron's veracity and experience across partitions of the MLP's domain. Then, using the veracity scores we order the MLP's domain based on the veracity measure. Finally, we compare the visualization of the ordered veracity and experience scores to Figures 8 and 9, respectively the ordering we desire for a classifier with good isolation and the ordering we expect from an arrogant classifier.

\section{Partitioning an MLP's domain into chambers}

Let $\mathcal{D}$ be two-class truthed data. Thus, $\mathcal{D}$ can be partitioned into two disjoint subsets $\mathcal{D}^{+}$and $\mathcal{D}^{-}$where $\mathcal{D}=\mathcal{D}^{+} \cup \mathcal{D}^{-}$ and $\mathcal{D}^{+} \cap \mathcal{D}^{-}=\emptyset$. A multilayer perceptron $F$ trained on the data $\mathcal{D}$ yields a hyperplane arrangement $A$. From this arrangement, one forms the associated chamber set Cham(A). Some of the chambers are populated with the " + " class data vectors; other chambers are populated with the "-" class data vectors. Some chambers-and, often, most-are not populated at all. If the MLP has done an excellent job of separating the data, then no chamber exists that contains both "-" class and "+" class vectors.

Let $C \in \operatorname{Cham}(\mathrm{A})$ be a chamber in the chamber set formed by the arrangement $A$. Based upon the truthed data $\mathcal{D}$, we can define three subsets of the chamber set $\operatorname{Cham}(\mathrm{A})$ as

$\operatorname{Cham}(\mathrm{A})^{+}=\left\{C \in \operatorname{Cham}(\mathrm{A}): x \in C\right.$ for some $\left.x \in \mathcal{D}^{+}\right\}$ $\operatorname{Cham}(\mathrm{A})^{-}=\left\{C \in \operatorname{Cham}(\mathrm{A}): x \in C\right.$ for some $\left.x \in \mathcal{D}^{-}\right\}$ $\operatorname{Cham}(\mathrm{A})^{u}=\operatorname{Cham}(\mathrm{A})-\left(\operatorname{Cham}(\mathrm{A})^{+} \cap \operatorname{Cham}(\mathrm{A})^{-}\right)$.

Since $\mathcal{D}^{+} \cap \mathcal{D}^{-}=\emptyset$, one hopes that the MLP has done an excellent job of separating the data into separates chambers, such that $\operatorname{Cham}(\mathrm{A})^{+} \cap \operatorname{Cham}(\mathrm{A})^{-}=\emptyset$. When the MLP has not separated the data then $\operatorname{Cham}(\mathrm{A})^{+} \cap \operatorname{Cham}(\mathrm{A})^{-} \neq$ $\emptyset$. Often, the MLP creates more chambers than needed and several chambers have no data in them; thus, in general, we have that

$$
\operatorname{Cham}(\mathrm{A})^{+} \cup \operatorname{Cham}(\mathrm{A})^{-} \neq \operatorname{Cham}(\mathrm{A}) .
$$

or the set of unpopulated chambers $\operatorname{Cham}(\mathrm{A})^{u} \neq \emptyset$.

\section{Ordering chambers by relative veracity}

Consider a single-hidden-layer multilayer perceptron constructed using hard-limiter sigmoids in the hidden layer nodes and tansig sigmoids in the output layer. Thus, given a chamber $C$ and a point $x \in C$, the MLP output yields a veracity $\mathfrak{v}(x) \in[-1,1]$ and, in fact, this veracity is the same value for all $x \in C$. Therefore, we consider the entire chamber $C$ and define the veracity of the chamber $\mathfrak{v}(C)=\mathfrak{v}(x)$. Since the space $\mathbb{R}^{d}$ is partitioned into the disjoint subsets of Cham $(\mathrm{A})$ by the MLP, then we need only to consider each chamber separately.

\section{Ordering chambers by relative experience}

We seek to identify each chamber $C \in \mathrm{Cham}(\mathrm{A})$ with a label representing relative experience. Define the experience mapping $\mathfrak{e}: \operatorname{Cham}(\mathrm{A}) \rightarrow\{t, f, i, e\}$. Populated chambers shall be identified as containing specific experience. Let the true label $t$ denote specific experience with "+"class, and let false label $f$ denote specific experience with "." class data. Unpopulated chambers shall be identified as either interpolations or extrapolations of training data. The interpolation label $i$ implies marginal experience in training data, and the extrapolation label $e$ implies insignificant-to-no experience with class data.

Our experience mapping $\mathfrak{e}$ utilizes a distance metric between chambers. For a simple metric, we only need to determine whether or not a chamber intersects any populated chamber. Let us construct a mapping that convenes this notion. Define the distance mapping $\sigma$ on chambers in $\operatorname{Cham}(\mathrm{A})$ to be

$$
\sigma\left(C^{\prime}, C^{\prime \prime}\right)=\left\{\begin{array}{lll}
0 & \text { if } & C^{\prime}=C^{\prime \prime} \\
1 & \text { if } & C^{\prime} \cap C^{\prime \prime} \neq \emptyset \\
2 & \text { if } & C^{\prime} \cap C^{\prime \prime}=\emptyset
\end{array}\right.
$$

for every $C^{\prime}, C^{\prime \prime} \in \mathrm{Cham}(\mathrm{A})$. We have the result that this mapping is a metric.

Theorem 1: The mapping $\sigma$ is a metric on Cham(A), and $(\mathrm{Cham}(\mathrm{A}), \sigma)$ is a metric space.

Proof: First of all, notice that $\sigma\left(C^{\prime}, C^{\prime \prime}\right)$ is defined for every possible choice of $C^{\prime}, C^{\prime \prime} \in$ Cham(A).

1. Clearly, $\sigma\left(C^{\prime}, C^{\prime \prime}\right) \geq 0$ for all $C^{\prime}, C^{\prime \prime} \in \operatorname{Cham}(\mathrm{A})$.

2. Clearly, $\sigma\left(C^{\prime}, C^{\prime \prime}\right)=\sigma\left(C^{\prime \prime}, C^{\prime}\right)$ for all $C^{\prime}, C^{\prime \prime} \in$ $\operatorname{Cham}(A)$.

3. Given $C^{\prime}, C^{\prime \prime}, C^{\prime \prime \prime} \in \mathrm{Cham}(\mathrm{A})$ then enumerating the different cases we see

$$
\sigma\left(C^{\prime}, C^{\prime \prime \prime}\right) \leq \sigma\left(C^{\prime}, C^{\prime \prime}\right)+\sigma\left(C^{\prime \prime}, C^{\prime \prime \prime}\right)
$$

4. If $C^{\prime} \neq C^{\prime \prime}$ then $\sigma\left(C^{\prime}, C^{\prime \prime}\right)=1$ or 2 , thus $\sigma\left(C^{\prime}, C^{\prime \prime}\right) \neq 0$ so $\sigma$ is positive definite.

Therefore, $\sigma$ is a metric defined on $\operatorname{Cham}(\mathrm{A})$. It follows that $(\mathrm{Cham}(\mathrm{A}), \sigma)$ is a metric space.

Now, suppose $\mathbb{C}$ is a subset of chambers in Cham(A). We define the distance from a chamber $C$ to a set of chambers $\mathbb{C}$ by

$$
\operatorname{dist}(C, \mathbb{C})=\min \left\{\sigma\left(C, C^{\prime}\right): C^{\prime} \in \mathbb{C}\right\}
$$

Observe that if $\operatorname{dist}(C, \mathbb{C})=0$ then chamber $C \in \mathbb{C}$.

We will chose special subsets for $\mathbb{C}$ among our populated chambers. Let us assume that $\operatorname{Cham}(\mathrm{A})^{+} \cap \operatorname{Cham}(\mathrm{A})^{-}=\emptyset$. 


\section{Vol. 5-2045}

Then, we can produce a mapping based upon the data set $\mathcal{D}=\mathcal{D}^{+} \cup \mathcal{D}^{-}$

$$
\mathfrak{e}(C)=\left\{\begin{array}{ccc}
t & \text { if } & \operatorname{dist}\left(C, \operatorname{Cham}(\mathrm{A})^{+}\right)=0 \\
f & \text { if } & \operatorname{dist}\left(C, \operatorname{Cham}(\mathrm{A})^{-}\right)=0 \\
i & \text { if } & \operatorname{dist}(C, \operatorname{Cham}(\mathrm{A}))=1 \\
e & \text { if } & \operatorname{dist}(C, \operatorname{Cham}(\mathrm{A}))=2
\end{array}\right.
$$

Thus, populated chambers are labeled either $t$ or $f$, and unpopulated chambers are labeled either $i$ or $e$.

\section{Mapping veracity versus expertise for the MLP}

Now associate with each chamber $C \in \mathrm{Cham}(\mathrm{A})$ the 2-tuple $(\mathfrak{v}(C), \mathfrak{e}(C))$. To visualize how the MLP will map every chamber (and thus every vector in $\mathbb{R}^{d}$ ) we generate all possible 2-tuples, by considering the set

$$
\{(\mathfrak{v}(C), \mathfrak{e}(C)): C \in \operatorname{Cham}(\mathrm{A})\}
$$

Since the chamber set Cham (A) is finite, we can order the chambers using their veracity values to get the ordered set

$$
\{\operatorname{Cham}(\mathrm{A}), \preccurlyeq\}=\left\{C_{1}, C_{2}, \ldots, C_{N}\right\}
$$

where $N=\operatorname{card}(\operatorname{Cham}(\mathrm{A}))$ and

$$
\mathfrak{v}\left(C_{1}\right) \leq \mathfrak{v}\left(C_{2}\right) \leq \cdots \leq \mathfrak{v}\left(C_{N}\right)
$$

Thus, we have defined the relation $\preccurlyeq$ on $\operatorname{Cham}(\mathrm{A})$ such that chamber $C_{i} \in \operatorname{Cham}(\mathrm{A})$ "precedes" chamber $C_{j} \in$ Cham(A) and write $C \preccurlyeq B$ if and only if $\mathfrak{v}\left(C_{i}\right) \leq \mathfrak{v}\left(C_{j}\right)$.

Now consider the set of 3-tuples

$$
\left\{\left(n, \mathfrak{v}\left(C_{n}\right), \mathfrak{e}\left(C_{n}\right)\right): n=1,2, \ldots, N\right\} .
$$

If the MLP $F$ isolates all the data perfectly, then the graph of this set yields plots like Figure 8. However, if the MLP F merely separates training data perfectly but arbitrarily orders extrapolated data, then the graph of the 3-tuple set yields plots like Figure 9.

\section{Resolving confusion in a solution to the Fisher iris problem}

For a specific illustration of an arrogant classifier, let us investigate an MLP solution for the Fisher iris classification problem. The Fisher iris dataset [10] represents 150 flowers, 50 each from 1 of 3 varieties of iris. Each flower is characterized by a vector capturing 4 numerical attributes. The data generalization we have selected to investigate is a multilayer perceptron with 14 hidden layer nodes and 3 output nodes trained via back propagation to separate the first class of iris from the other two classes. This MLP is an interesting solution as our investigation has revealed that, though the MLP does not appear to memorize the data, the solution still represents a poor generalization of the Fisher iris problem because the MLP generates a significant number of arrogant classifications.
For the MLP, we were given the following: the weights and biases of a single-hidden layer perceptron, the two datasets used to train and evaluate the MLP, and the error curve generated in the training of the MLP. We were not privy to the training of this network, but we were supplied with the final parameter set of the design - the hidden layer weights $\left[\bar{W}_{h}: \bar{b}_{h}\right]$ and the output weights $\left[\bar{W}_{o}: \bar{b}_{o}\right]^{T}=\left[u_{1}, u_{2}, u_{3}\right]$.

$\bar{W}_{h}: \bar{b}_{h}={ }$

$\left[\begin{array}{rrrrr}0.2599 & 0.3348 & -0.3876 & -0.2779 & 0.4585 \\ -0.3184 & 0.1027 & 0.3266 & -0.3127 & -0.1405 \\ -0.2844 & -0.0509 & -0.0714 & 0.3860 & 0.0187 \\ 0.1902 & 0.1249 & -0.3105 & -0.3983 & -0.0660 \\ -0.1328 & 0.6630 & 0.0140 & 0.3430 & 0.3249 \\ -0.2037 & 0.1119 & 0.0675 & -0.0988 & 0.1614 \\ 0.3562 & -0.2434 & -0.3651 & -0.3105 & 0.1878 \\ -0.4330 & 0.1389 & -0.1011 & -0.3456 & 0.5038 \\ -0.0740 & -0.2961 & -0.1801 & 0.2190 & -0.0622 \\ 0.2210 & -0.1963 & -0.3508 & -0.0272 & 0.0245 \\ -0.3331 & -0.4145 & 0.1865 & 0.1978 & -0.3176 \\ 0.1955 & -0.5565 & -0.2773 & -0.6217 & -0.5234 \\ 0.6625 & 0.4879 & -0.6841 & -0.1655 & 0.0317 \\ -0.1643 & 0.1604 & 0.2078 & 0.1187 & -0.0467\end{array}\right]$

$$
\left[\bar{W}_{o}: \bar{b}_{o}\right]^{T}=\left[\begin{array}{rrr}
2.8781 & 17.3599 & 3.2325 \\
3.8707 & -20.5670 & -12.0076 \\
0.9027 & -15.2658 & -12.6445 \\
6.5404 & -40.9967 & -7.3975 \\
1.1803 & -21.1980 & 9.7689 \\
2.0187 & -17.7602 & -4.9986 \\
0.7589 & -4.0878 & -0.9049 \\
2.4011 & 40.4554 & -20.8312 \\
0.5458 & 3.1439 & -11.4082 \\
1.0027 & -4.8418 & -6.2945 \\
0.7954 & 9.6175 & -18.5234 \\
4.9099 & -8.9478 & -0.7643 \\
3.5889 & 19.2883 & -39.8599 \\
-0.6176 & 3.6268 & 3.6068 \\
-4.6405 & -16.0082 & -25.2557
\end{array}\right]
$$

First, let us investigate whether or not the neural network is memorizing. It is simple to show using principle component analysis [11] that the problem of separating the first Fisher iris class from the other two classes is a linear problem. Thus, a successful solution to the problem requires only a single perceptron. Since the MLP under investigation uses a 14-hidden-node architecture, rules of thumb [12] tell us that the solution is unnecessarily complex and the MLP has likely memorized the training data. However, an analysis of the populated chambers and unpopulated chambers of interest belies this intuition. Instead of constructing an error matrix, we use our method [13] of resolving populated chambers. Figures 10 and 11 shows the results: out of a possible 1471 chambers, only 4 chambers are populated with training data and 5 chambers (the 4 populated chambers and one additional chamber) contain evaluation data. From Figure 11, we see little evidence of memorization. Only a single evaluation exemplar fell outside of the 4 populated chambers; and, 


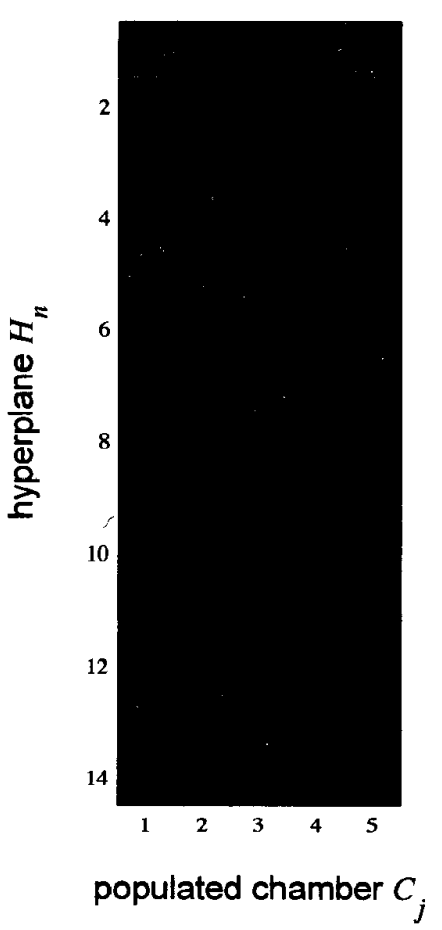

Figure 10. The populated chambers for a multilayer perceptron trained by back propagation to solve the Fisher iris problem. The first four chambers were the chambers populated by both the training set and test set. The fifth chamber was populated by a single datum from the test set.
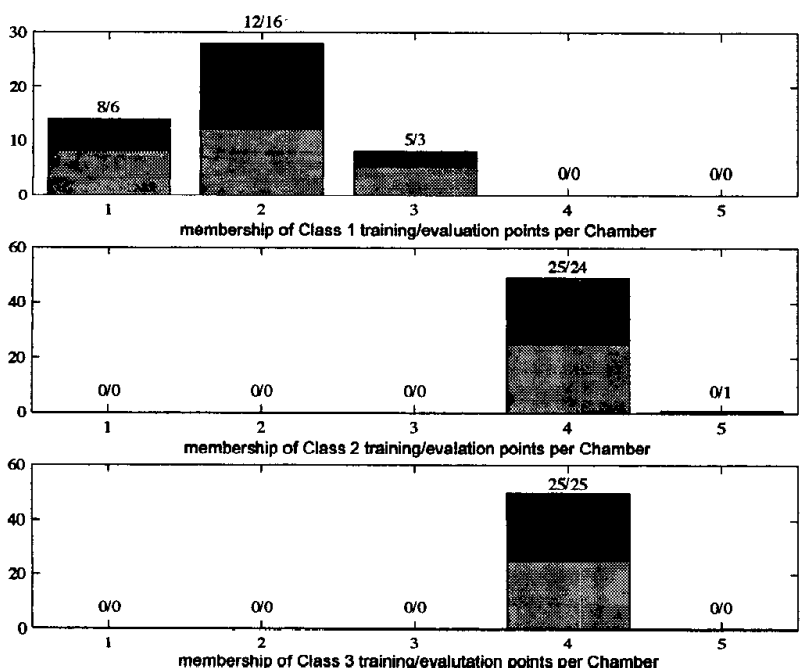

Figure 11. The membership of the populated chambers by class. The bar charts represent the sum of the membership by training set (lower) and test set (upper) respectively.

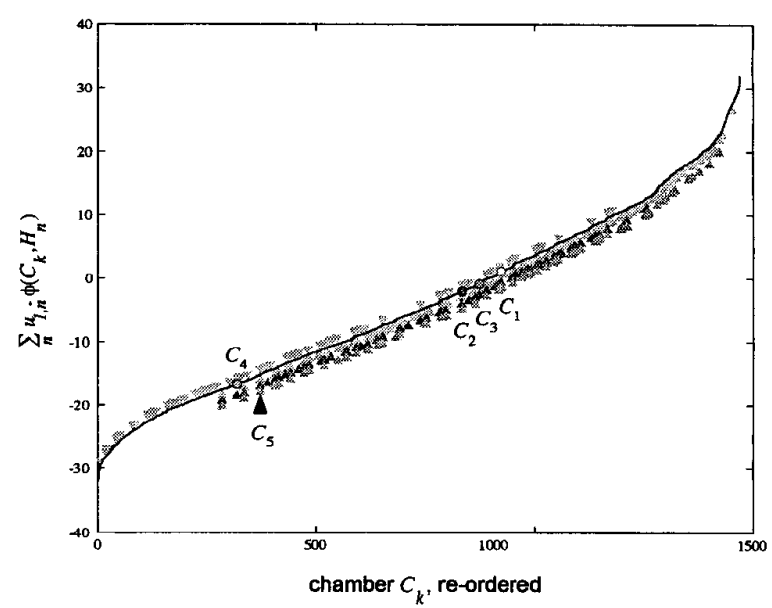

Figure 12. Weighted ordering of chambers in the Fisher iris feature domain via output node 1 of MLP $F$.

though it is arguably excessive to separate Class 1 data into 3 separate chambers, the ratio of training data to evaluation data membership in each of these chambers is relatively even.

So, the evidence for memorization in this particular MLP solution is thin. Now, let us consider arrogance. Recall, in Section 2 we asserted that a good generalization may be illustrated by ordering the feature set of a classifier in terms of its veracity scores and, then, cross referencing this ordering to the classifier's experience scores. Further, we defined measures to specify the veracity and experience scores over the domain of an MLP given the network's weights and biases and the data used to train the network. Now, we have applied these measure to the 3 -output-node MLP solution above and generated Figures 12,13 and 14 to show the domain ordering specified by each output node. We used unnormalized veracity scores to simplify the calculation of the plots as they are merely scaled and shifted versions of the normalized graphs. In each plot, a point on the $S$-curve specifies a chamber. Specially notated chambers include populated chambers (denoted as circles labeled $\left.C_{1}, C_{2}, C_{3}, C_{4}\right)$, the unpopulated chamber of interest (the larger triangle labeled as $C_{5}$ ), and unpopulated chambers labeled as interpolations with experience scores of $\mathfrak{e}(C)=i$ (smaller triangles). The remaining points are unpopulated chambers labeled as extrapolations, i.e., designated an experience score $\mathfrak{e}(C)=e$. Note in each graph, the veracity scores of populated chambers are not ordered at the extremes of the S-curve but instead along the linear portion of the curve. Extrapolated chambers appear arbitrarily along the entire length of the curve. These graphs each reflect a significant number of arrogant classifications with Figure 14 representing the worst offender, output node 3 .

We have demonstrated a strong example of arrogance here. In further empirical experiments, we have found that the ordering of the chamber set implemented by a multilayer perceptron trained by back propagation rarely achieves the or- 


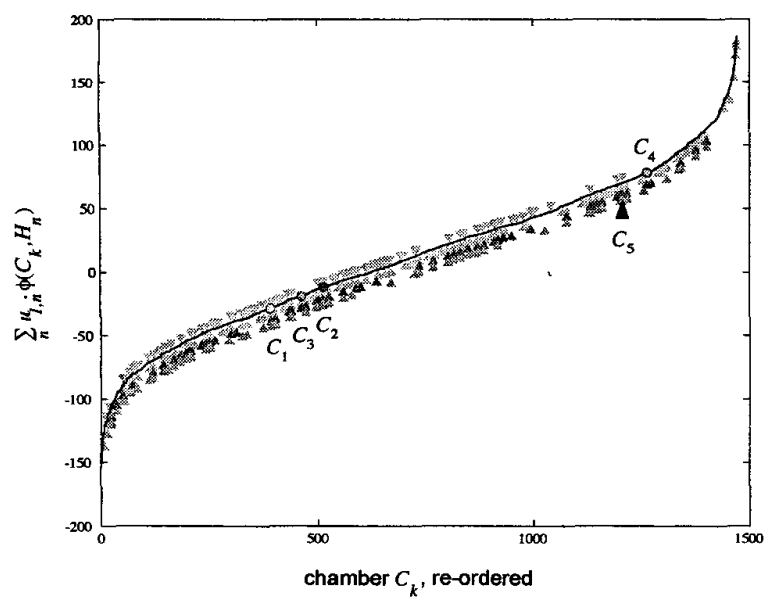

Figure 13. Weighted ordering of chambers in the Fisher iris feature domain via output node 2 of MLP $F$.

dering of a good generalization shown in Figure 8. Instead, the expected veracity is more closely reflexed in Figure 9-a result that means that the classifier generates arrogant classifications. A multilayer perceptron that maps in this way gives interpolated and extrapolated regions stronger veracity scores than regions containing true and false training data.

When applied to the multilayer perceptron, the expectation of veracity shown in Figure 9 assumes that (1) the hyperplane arrangement A implemented by the MLP does not define a convex hull, (2) the complement of the hyperplane arrangement A (formed by reversing the sign on all weights and biases) also does not define a convex hull, and (2) the chambers that are assigned as true, false and interpolated are bounded, i.e., chambers of finite volume. These are reasonable assumptions given $d \ll N$ where $d$ is the dimension of the feature set and $N$ is the number of first-hidden-layer nodes. The expectation also relies on the assumption that back propagation tends to iterate to an MLP solution where the hyperplane arrangement of the first hidden layer is not geometrically symmetric.

\section{Benefits of 4-value logic method}

Benefits of our 4-value logic technique over other iterative and stochastic methods include (1) model selection, (2) data presentation, and (3) domain coverage. Iterative methods such as cross validation and bootstrapping require that the evaluation of generalization take place over multiple training runs ${ }^{3}$ prior to model selection. These iterative techniques serve to prevent memorization (and memorization only); at best, they are appropriate for selecting an architecture for po-

\footnotetext{
${ }^{3}$ In each run, the training dataset, or augmented dataset, is split into two: one subset is used to train a solution iteratively, and the second subset is used to calculate the classification error for each training epoch. Training of the current model continues until the classification error stabilizes and then starts to increase. In subsequent runs, a new solution is trained using a different subset of data. The classification error curve calculated for each run is fused with the other curves to form an estimate of generalization "error". [14]
}

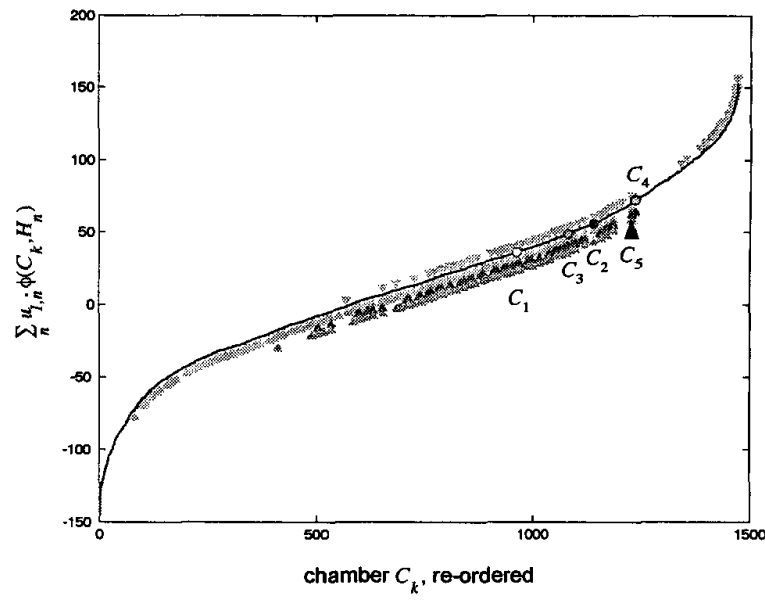

Figure 14. Weighted ordering of chambers in the Fisher iris feature domain via output node 3 of MLP $F$.

tential solutions, not for identifying the solution with the best potential. In cross validation and bootstrapping, model selection is delayed until after the generalization evaluation is complete and many of subsequent solutions have been discarded arbitrarily. Once a model is selected, the estimation of generalization for the model itself is optimistic. In contrast, our technique evaluates the generalization of an MLP after model selection. The evaluation requires only the classifier's weights, biases and the data used to train these parameters. Our technique demonstrates the weaknesses of a specific solution via deterministic measures-not through estimatesuncovering both problems in both memorization and arrogance if they exist.

One test of the effectiveness of a generalization evaluation technique is its sensitivity to data presentation. A good technique should not be sensitive to changes in the order that training data are presented. Estimates should vary with changes to the membership of the training set, but not to mere changes in order of presentation. It should be a red flag that cross validation and bootstrapping demonstrate sensitivity to data presentation. The results of our evaluation do not change based on the order that data are presented. This is because our technique is based on the membership of chambers, and a training datum-no matter its order in the training set-is either a member of a particular chamber or it is not. This leads to much more consistent estimates of generalization based directly on the training set used to generate a particular solution and unbiased by alternative training sets used to generate alternative solutions not currently under consideration.

Another simple test of the effectiveness of a generalization evaluation technique is its domain coverage. We assert that, if the measure of the hypothesized operational set is positive, then the measure of domain coverage for a generalization evaluation should also be positive and preferably at least equal to the measure of the hypothesized operational set. 
Stochastic techniques exercise very little use of the domain, that is, the measure of the set of feature vectors used in the evaluation of generalization is zero. For our technique, the measure of domain coverage is positive; in low-dimensional cases such as the Fisher iris problem above, we are able to exercise the entire domain.

\section{CONCLUSION}

Arrogance in classification is likely to occur when training criteria do not require the isolation of class data, merely the separation of class data. In order to properly regulate generalizations between the extremes of memorization and arrogant classification, evaluations must take confusion measures in consideration in addition to traditional error measures. The goal of expertise logic and 4-value logic is to construct expert classifiers that realistically model experience across an entire domain. We have presented the means to succinctly view the ordering imposed by a multilayer perceptron over a feature set in terms of 4-value logic. This representation of (Class(Cham), $\preccurlyeq)$ demonstrates the arrogant expressions of the multilayer perceptron and allows for informed manipulation of the ordering over both populated and unpopulated disjoint subsets to improve data generalization.

\section{REFERENCES}

[1] Amy L. Magnus, Inquisitive pattern recognition, Ph.D. dissertation, Air Force Institute of Technology, 2003.

[2] David John Chalmers, The Conscious Mind: In Search of a Fundamental Theory, Oxford University Press, New York, 1997.

[3] Alan M. Turing, "Computing machinery and intelligence," Mind, vol. 59, no. 236, pp. 433-460, 1950.

[4] Steven K. Rogers, Matthew Kabrisky, Kenneth Bauer, and Mark E. Oxley, "Computing machinery and intelligence amplification," in Proceedings of 2002 World Congress on Computational Intelligence, Honolulu, HI, May 2002.

[5] Encarta World English Dictionary, Bloomsbury Publishing, 1999.

[6] Amy L. Magnus and Mark E. Oxley, "The theory of confusion," in Proceedings of SPIE, Applications and Science of Neural Networks, Fuzzy Systems, \& Evolutionary Computation IV, San Diego, CA, August 2001, pp. 105-116.

[7] Stephen G. Alsing, Kenneth W. Bauer, Jr., and John. O. Miller, "A multinomial selection procedure for evaluating pattern recognition algorithms," Pattern Recognition, to appear 2002.

[8] Tom M. Apostol, Mathematical Analysis, AddisonWesley, Reading, MA, second edition, 1974.

[9] Amy L. Magnus and Mark E. Oxley, "Quantifying the expertise of classifiers using 4-value logic," in Proceedings of SPIE, Applications and Science of Compu- tational Intelligence V, Orlando, FL, April 2002, vol. 4739.

[10] R. A. Fisher, "The use of multiple measurements in taxonomic problems," Annals of Eugenics, vol. 7, pp. 179-188, 1936.

[11] Rafael C. Gonzales and Richard E. Woods, Digital Image Processing, Addison-Wesley, Reading, MA, 1992.

[12] Lemuel Ray Myers, Radial complexity estimation for improved generalization in artificial neural networks, Ph.D. dissertation, Air Force Institute of Technology, 1998.

[13] Amy L. Magnus and Mark E. Oxley, "Generalization tools for multilayer perceptrons," in Proceedings of 2002 World Congress on Computational Intelligence, Honolulu, HI, May 2002.

[14] Christopher M. Bishop, Neural Networks for Pattern Recognition, Clarendon Press, Oxford, 1995.

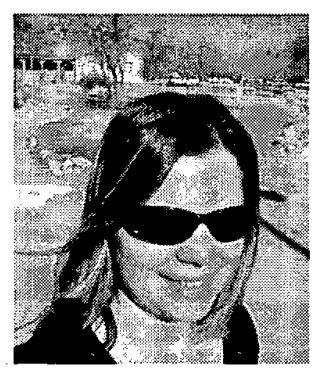

Major Amy L. Magnus was commissioned into the United States Air Force in 1990, the same year she received the B.S. degree in electrical engineering from the Rochester Institute of Technology, Rochester, NY. She received her M.S.E.E. degree in 1995 from the Air Force Institute of Technology (AFIT), Wright-Patterson AFB, OH. She successfully defended her dissertation in October 2002 and shall walk through her hooding ceremony March 2003 receiving the Ph.D. degree in electrical engineering from AFIT. Currently, she is a program manager at the Defense Threat Reduction Agency overseeing research efforts in information assurance and fault tolerance for low-powered integrated circuits, devices sensitive to single event upset.

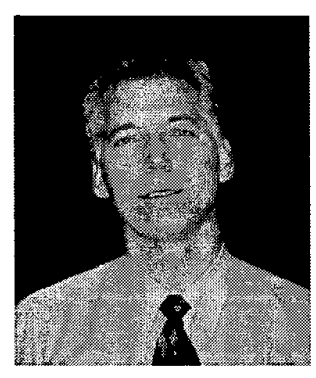

Dr Mark E. Oxley received the B.S. degree in mathematics in 1978 from Cumberland College, Williamsburg, KY, the M.S. degree in applied mathematics in 1980 from Purdue University, West Lafayette, IN, and the Ph.D. degree in mathematics in 1987 from North Carolina State University, Raleigh, NC. Since 1987, he has been with the Graduate School of Engineering and Management at the Air Force Institute of Technology, Wright-Patterson Air Force Base, $\mathrm{OH}$, where he is a Professor of Mathematics in the Department of Mathematics and Statistics. His current research interests include classifier fusion, artificial neural networks, wavelets analysis, functional analysis, optimization and nonlinear partial differential equations. 